\title{
La cartografía de la movilidad: la diáspora en el marco de la articulación entre lo local y lo global' ${ }^{1}$.
}

\section{Lia Rodriguez de la Vega ${ }^{2}$}

Artículo científico

Material original autorizado para su primera publicación en el Journal de Ciencias Sociales, Revista Académica de la Facultad de Ciencias Sociales de la Universidad de Palermo.

Recibido: 1-11-2013

Aceptado: 25-2-2014

\section{Resumen}

La diáspora india, una de las mayores del mundo, cuenta de acuerdo a los datos provistos por el Comité de Alto Nivel sobre la Diáspora India en su informe, con más de 20 millones de indios que residen fuera de la India, asentados en más de 70 países con concentraciones de diverso volumen en ellos (MEA, 2002).

Sheffer (1986) señala que la relación triádica entre el país de origen-la diáspora y los países de residencia deviene un aspecto integral e incluso permanente del flujo de política nacional e internacional. Esa relación da cuenta de dinámicas que articulan lo local y lo global y que abarcan numerosas cuestiones, entre ellas: las políticas desarrolladas por el estado de origen hacia la diáspora, sus implicancias para las relaciones bilaterales y multilaterales, cuestiones relativas a la ciudadanía, la réplica/continuación de construcciones políticas locales indias en países de asentamiento, la implicación de los emigrados indios en cuestiones socio-políticas de los lugares de residencia, el desarrollo de diversos intereses económicos y todo ello implica también y fundamentalmente, cuestiones identitarias, niveles de influencia y recursos (Newland, 2010).

Así, el presente trabajo realiza una aproximación a la diáspora india en el marco de la articulación entre lo local y lo global, concluyendo que la enunciación misma de la "diáspora india", desde la perspectiva considerada, da cuenta ya de una articulación que por lo demás es una tarea permanente en el contexto del significado/la resignificación identitaria de la "indianidad", sus alcances sobre la configuración del espacio y el tiempo, y la capacidad de influencia que ello conlleva.

Palabras clave: Diáspora india, articulación local- global, identidad, configuración espacio temporal.

\section{Abstract}

The Indian diaspora, one of the largest ones in the world, is estimated at over 20 million Indians abroad, living in more than 70 countries with different volume of population in them, according to the High Level Committee on Indian Diaspora's Report (MEA, 2022).

\footnotetext{
${ }^{1}$ El término "cartografía" alude aquí al "mapeo/ubicación" espacio - temporal de los emigrados indios en la construcción que de ellos hacen otros agentes sociales, que los sitúan como parte de un "nosotros" u "otros". Esta ubicación puede potenciar la proyección del país de origen en los distintos países de residencia de sus emigrados y permitir a los emigrados fortalecer un sentido de "localidad" con el país de origen.

${ }^{2}$ Dra. en Relaciones Internacionales. Profesora Titular Facultad de Ciencias Sociales, Universidad de Palermo. Miembro del CARI. Profesora Adjunta Facultad de Ciencias Sociales, Universidad Nacional de Lomas de Zamora.
} 
Sheffer (1986) points out that the triadic relationship among the country of origin, the diaspora and the country of residence becomes an integral and even permanent aspect of national and international politics. This relationship shows dynamics that articulate the local and the global and they include diverse subjects such as: the government's politics of the country of origin towards the diaspora, its implications for bilateral and multilateral relationships, different issues about citizenship, the replication of local Indian political construction in the countries of residence, the implication of Indian emigrants in socio political issues in the countries of residence, the development of different economic interests which also imply especially identity issues, levels of influence and resources (Newland, 2010).

Thus, this article is an approximation to the diaspora in the context of the articulation between local and global, ending with the idea that the mere enunciation of the diaspora, from the considered perspective, shows an articulation, and this articulation is a permanent work, in the context of the identity meaning/re-elaboration of the meaning of "Indianness", its scope on the configuration of space and time and the capacity of influence that this implicates.

Key Words: Indian diasporas, local-global articulation, identity, space/time configuration

\section{Introducción}

El trabajo que presentamos se propone una aproximación a la diáspora india en el marco de la articulación entre lo local y lo global, atendiendo al significado/la resignificación identitaria de la "indianidad", sus alcances sobre la configuración del espacio y el tiempo, y la capacidad de influencia que de ello deriva. Consideramos distintos autores que han abordado esta temática, otras relacionadas y el reporte que sobre la diáspora india hiciese el Comité de Alto Nivel sobre la diáspora India comisionado para ello (MEA, 2002).

\section{La diáspora india}

Cohen (Vertovec y Cohen, 1999) señala que el término diáspora asociado a la dispersión forzosa se encuentra ya en el Deuteronomio, ligado inicialmente a la historia religiosa de judíos y cristianos $^{3}$. El uso disciplinario del término fuera de las asociaciones mencionadas, es atribuido por Bauman (2001) a George Shepperson en referencia a la diáspora africana, en 1965, y a partir de allí, el estudio de las diásporas devendría progresivamente un campo de estudio.

De acuerdo a Leclerc (2004), el uso de la idea de una "diáspora" para aludir a los emigrados de la India, aparece en un artículo de Bharati (1976) sin que el término se mencionara de manera explícita. Agrega que, si bien Jain (1993) aludió a las comunidades indias en el extranjero también remarcó su diversidad, mientras que Sharam (1989) se refirió a "los indios en el extranjero" y ya en 2004, Jayaram hizo pleno uso del término "diáspora". Ese reconocimiento de la categoría "diáspora india" se vería reforzado por la realización de conferencias internacionales que abordaron la temática, siendo antecedidas por la creación de un centro para el estudio de esta diáspora, en la Universidad de Hyderabad, India, en $1996^{4}$.

\footnotetext{
${ }^{3}$ Como veremos inmediatamente, el uso del término "diáspora" asociado a una connotación negativa, permaneció como predominante hasta que el alcance semántico del término se extendió (Cohen, 1997).

${ }^{4}$ Leclerc (2004) menciona también otros términos utilizados en asociación a la idea de la diáspora india: indios allende los mares" o
} 
Oonk (2007) menciona tres perspectivas sobre el concepto de diáspora: a) la que alude al marco analítico de los académicos ${ }^{5}$, b) la sostenida por el gobierno de la India, que define a la "diáspora india" (2002) como "un término genérico que describe personas emigradas de territorios que están actualmente dentro de los límites de la República de la India. Se refiere también a sus descendientes" y señala que estas personas "residentes en tierras lejanas" "retuvieron su ligazón emocional, cultural y espiritual con su país de origen"6 y c) la perspectiva de los migrantes mismos. Es la segunda perspectiva la que nos ocupa en este trabajo, aunque cabe señalar que al igual que el autor, consideramos que hablar de "diáspora india" supone una primera mirada crítica de ambos términos en razón de cuestionar a qué refieren exactamente, qué tipo de unidad suponen y desde quién son enunciados ( $y$ construidos).

La "invención" de la diáspora india en particular7 (término cuyo uso académico se popularizó en la década del '90) estuvo signada y se difundió en gran medida con/por la creación, por parte del gobierno indio, del Comité de Alto Nivel sobre la Diáspora India (2000), que realizó un relevamiento de las comunidades expatriadas, categorizando a las personas al interior de la misma como veremos inmediatamente. Su definición de la diáspora señala no solamente que los emigrados que considera han mantenido su identidad india (sin definir la misma), sino que al mismo tiempo permite establecer una continuidad histórica entre todos los lugares de asentamiento y el país de origen.

Ya en la consideración del contexto globalizado, Khanna (2005) sostiene que la diáspora india, en vez de permanecer como geográficamente fragmentada, ve hoy la posibilidad de existir como un espacio imaginado y compartido, señalando que "Bollystan", el cosmopolitismo indio, es plantado en todos lados y emerge orgánicamente como una mixtura entre el etno -comercio y un nuevo modelo de trascendencia de la soberanía geográfica, expresando cabalmente la operación acontecida.

Como señaláramos, la diáspora india cuenta con más de 20 millones de indios que residen fuera de su país de origen incluyendo: a los ciudadanos indios que no residen en India (Nri's ${ }^{9}$; a

\footnotetext{
"indios de ultramar", "East Indian", "Asian Indian", "South Asian", "asiático" Otros usados en el marco de un nacionalismo cultural son: Bharatya (indio), atendiendo a la dispersión de los hijos de Bharat Mata; Bharatavasi (habitante actual de la India), y Bharatvanshi (descendiente de un habitante de la India).

${ }^{5}$ En este sentido, cabe mencionar que al hablar de diáspora, Vertovec señala que este término es usado actualmente para describir prácticamente cualquier población considerada "desterritorializada" o "transnacional" (esto es, surgida en una tierra distinta de aquella en la que reside y cuyas redes socio-políticas y económicas atraviesan los límites de naciones- Estado o se expanden por el mundo). (Vertovec y Cohen, 1999)

${ }^{6}$ The Indian Diaspora, párrafo 1 y 2

7 La "invención" no alude a cuestionar el uso del término "diáspora" mismo, sino a situarlo en razón de que el uso de determinada terminología para referirse a un grupo social para reconocerlo como tal, contribuye a investirlo de determinados sentidos. Las clasificaciones distinguidas al interior de esta "diáspora" (que en líneas generales subsume "las diásporas", en términos de las heterogeneidades al interior de esta emigración, aunque en el discurso oficial esas diferencias se reconocen) contribuyen a regular las relaciones entre el país de origen y sus emigrados (ahora considerados "la diáspora india"). Diáspora, NRIs y PIOs, son así categorías que permiten ligar no solamente el país de origen a los emigrados, sino la identidad india al ámbito transnacional (recuperando las maneras de "ser indio", residiendo fuera de India) y mediante este investidura o inscripción social, se habilita también a los indios que residen en India, a pensarse como parte de un espacio nacional extendido. Al respecto, puede consultarse Amrute (2012) y Varadarajan (2010), entre otros.

${ }^{8}$ El autor allude a este término del siguiente modo: "Bollystan was a mix of Bollywood, which is the way in which people think about Indian culture, as this all borders on composing, if culture essence that is Indian, and it's everywhere. And stan is just the suffix that means country. And to me, Bollystan is this global Indian culture that knows no borders, and is everywhere were the Diaspora is, and even where it isn't, it sort of infects audiences and gets people watching Bollywood. But it wasn't really just it up Bollywood. It was Indianness more broadly; an Indian culture and the ways in which India can project itself as a global presence, even if it isn't the global economic or strategic heavy weight. And that, Bollystan, would in a way, be a pillar of that strategy of making India a great power, even when it isn't" (Khanna, 2008, [párrafo 1-3]).
}

${ }^{9} \mathrm{Nri}$ (Indian citizens not residing in India). 
las personas de origen indio que han tomado otra nacionalidad (PIO's ${ }^{10}$ ); y a las personas de origen indio que legal y técnicamente pueden calificarse como personas apátridas de origen indio, que no cuentan con documentación que permita acreditar su origen indio (Sharma, s.f.).

La diáspora se localiza en más de 70 países, superando el millón de personas en once países (entre ellos, Sudáfrica, Estados Unidos de América, Reino Unido, etc.) y otros en que constituyen grupos de por lo menos cien mil personas, como Nepal, Singapur, Kenia, Omán, etc. (MEA, 2002). Xu (2009, citado en Hercog y Sieggel, 2010) señala que los diez países que más inmigrantes de este origen poseen son: Ios Emiratos Árabes Unidos, Arabia Saudita, Estados Unidos de América, Bangladesh, Nepal, Reino Unido, Sri Lanka, Canadá, Kuwait y Omán.

Se puede distinguir, a grandes rasgos, tres oleadas migratorias principales, atendiendo a la historia y la economía política india:

1) la emigración que comenzó alrededor de 1830, bajo el colonialismo europeo, dirigida hacia distintas colonias.

2) la emigración hacia los países desarrollados industrializados, durante el período de postguerra de la Segunda Guerra Mundial.

3) la emigración reciente a Asia occidental (Bhat, 1998).

\section{Las políticas del país de origen}

Mientras la lucha anticolonial encontró a la India cercana a sus migrantes, siendo la figura central de tal lucha Gandhi, él mismo un emigrado a Sudáfrica, con el partido del Congreso defendiendo los intereses de los emigrados ante situaciones diversas, Nehru no cultivó esos lazos en razón de una política de no intervención ${ }^{11}$, continuada por Indira Gandhi. El gobierno Janata ${ }^{12}$, en 1977, inició algunos cambios favorables hacia los emigrados y Rajiv Gandhi, tras su llegada al poder en 1984, siguió abriendo camino hacia ellos, atento a la relevancia del potencial económico que ellos significaban para la India ${ }^{13}$, actitud que se vería profundizada junto con las reformas económicas de liberalización de la década del ‘90. Y fue el gobierno de Atal Bihari Vajpayee (Partido Bharatiya Janata) el que llevó adelante un giro importante en la relación del gobierno indio con los emigrados ${ }^{14}$ (Leclerc, 2004; Hercog y Siegel, 2010) cuyo derrotero continúa en la actualidad.

\footnotetext{
${ }^{10}$ PIO's (Persons of Indian Origin who have acquired the citizenship of some other countries). De acuerdo a Sharma (s.f.) pueden incluirse aquí las siguientes categorías: 1) personas que en algún momento tuvieron un pasaporte indio; 2) personas cuyos padres, abuelos o bisabuelos hayan nacido y residido permanentemente en India, de acuerdo con lo estipulado en el Acta del Gobierno Indio (1935), o bien en otros territorios que más tarde se volverían parte de India; siempre y cuando esta persona no haya sido ciudadana de los países mencionados en el párrafo 2 (b) de la notificación núm. 26011/4/98-IC.1 de la mha, con fecha del 30 de marzo de 1999 ; 3 ) los cónyuges de ciudadanos de la India o de personas de origen indio que cumplan con las características de las dos categorías de PIO mencionadas anteriormente.
}

${ }^{11}$ En ese contexto, se recomendó a los emigrados adquirir la ciudadanía local y trabajar por la independencia en su lugar de residencia actual (Lal, 2001, citado en Nijhawan, 2003/2004). Nehru expresó, dirigiéndose al Lok Sabha, que India se interesaba por sus emigrados en razón de su historia común pero como habían podido optar entre la ciudadanía india y la del lugar de residencia, para aquellos que hubiesen tomado la segunda opción, "we have no concern with them [...because] politically they ceased to be Indians" (Nehru, 1961, citado en Nijhawan, 2003/2004).

12 Cabe recordar aquí que Atal Bihari Vajpayee fue Ministro de Asuntos Exteriores de esta gestión y sería posteriormente Primer Ministro, con el triunfo del Partido Bahratiya Janata.

${ }^{13}$ Durante su gestión, se creó, en 1986, una Célula Especial, en el ámbito del Ministerio de Finanzas, para atender a las inversiones de la diáspora.

${ }^{14}$ Los emigrados indios pasaron, como bien señalan Sinha- Kerkhoff y Bal (2003), de ser considerados "Non-required Indians"/ "Not Really Indian" (NRI) a Mother India's Children". 
En el marco global, la población emigrada empezó a ser sujeto de otra mirada desde la gestión de gobierno en India y atendiendo a ello, él mismo tomó cada vez más acciones en torno a la diáspora, a saber: la puesta en marcha del Plan de Credenciales para las PIO's que consideraba todos los segmentos de la diáspora (1999); la creación de una División de NRI's y PIO's, perteneciente al Ministerio de Asuntos Exteriores, presidida por un secretario; el nombramiento/creación de un Comité de Alto Nivel sobre la Diáspora India que realizó un informe exhaustivo de la diáspora india y su relación con India, concluyendo con diversas recomendaciones (2000); el establecimiento del Pravasi Bharatiya Divas (Día de la Diáspora India), el 9 de enero, recordando el regreso de Gandhi de su estadía en Sudáfrica y la aceptación de la petición de doble ciudadanía para algunos países de residencia, vigente desde $2003^{15}$, la decisión de fundar el Pravasi Bharatiya Kendra (el Centro de la Diáspora India) en Nueva Delhi (2004) y la posterior creación del Ministerio para los Asuntos de los Indios en el Extranjero (MOIA), a cargo de un ministro autónomo. También, pueden mencionarse diversos programas como el Programa de Becas para los Niños de la Diáspora (SPDC) que ofrece becas de grado en universidades indias abierto a NRI's y PIO's de 40 países; el Programa "Conoce India”; el Programa "Buscando las Raíces"; etc. (Ashraf, 2005; Hercog y Siegel, 2010).

Las iniciativas del Ministro en años recientes se refieren a reformas legislativas, regulatorias, procesuales y de cooperación internacional, y su mandato puede resumirse en tres cuestiones fundamentales: a) el desarrollo de redes con y entre los emigrados; b) el empoderamiento y protección de los mismos; y c) la promoción y facilitación del comercio e inversiones en la India, por parte de esos emigrados.

Existen distintas instituciones que colaboran en la implementación de la tarea del Ministro:
a) El Centro de Facilitación de los Indios de Ultramar
b) El Consejo Indio de Empleo de Ultramar
c) El Consejo Asesor Global en la Oficina del Primer Ministro
d) La Fundación India para el Desarrollo de los Indios de Ultramar
e) La Red Global India de Conocimiento
f) El Centro de los Indios de Ultramar

Las reformas en la administración del fenómeno migratorio han tenido/están teniendo lugar en los planos nacional, bilateral y multilateral.

El Ministerio atiende las recomendaciones expresadas por el Comité de Alto Nivel sobre la diáspora india acerca de las dificultades de los emigrantes indios en la zona del Golfo ${ }^{16}$ y en razón de ello, se crearon distintas instancias institucionales como: el Consejo Indio de Empleo para los emigrantes, Fondos para Bienestar de la Comunidad India, el Centro de Recursos para Trabajadores Emigrados, un Centro de Recursos para Migrantes (Kochi, Kerala), el Protector General de Emigrantes (que supervisa el desempeño de las agencias de reclutamiento de trabajadores), etc. (Hercog y Siegel, 2010).

\footnotetext{
${ }^{15}$ Los beneficiarios de esta doble ciudadanía (PIOs de Estados Unidos, Canadá, Reino Unido, Países Bajos, Italia, Irlanda, Portugal, Suiza, Grecia, Chipre, Israel, Australia, Nueva Zelanda, Francia, Suecia y Finlandia) consiste en que pueden invertir en la industria y en la agricultura, adquirir propiedades, enviar a sus hijos a escuelas y universidades pero no tienen el derecho a votar o a postularse para la función pública (The Indian Diaspora, s. f.; Chemouni, 2009). Atendiendo a los lugares de residencia de aquellos que pueden solicitarla, Leclerc (2004) comenta que se habla de un "apartheid de dólares".

${ }^{16} \mathrm{Al}$ respecto, se puede consultar Khadria (2006).
} 
Se han firmado también tratados bilaterales con diversos países (Qatar, en 1985; Jordania, en 1988; Emiratos Árabes Unidos, en 2006; Kuwait en 2007; Omán, en 2008 y Malasia y Bahrain, en 2009. Se firmó un Memorando de Entendimiento sobre Asociación concerniente a Movilidad Laboral, con Dinamarca y se busca avanzar en instancias similares con otros países europeos y surasiáticos. En 2006, se firmó el primer acuerdo bilateral de seguridad social con Bélgica y luego con los Países Bajos, Alemania, Francia, Luxemburgo, Dinamarca, Hungría, República Checa y Suiza, iniciándose las gestiones para avanzar en acuerdos similares con Estados Unidos de América, Reino Unido y Canadá.

En el ámbito multilateral, India participa del Proceso Colombo ${ }^{17}$, apoyado por la Organización Internacional para las Migraciones (OIM). También se firmó un memorando de Entendimiento con la OIM, acordando condiciones para la mejor administración de empleo legal de trabajadores indios en la Unión Europea. En razón de la creciente relevancia de la emigración india calificada hacia Europa, se observa un aumento de tareas de cooperación (existe desde 2000, un grupo de trabajo conjunto India-Unión Europea sobre asuntos consulares y se adoptó, en 2005, un Plan Estratégico de Acción Conjunta). Paralelamente, India es un miembro activo del Encuentro Asia-Europa (ASEM) ${ }^{18}$ y participa de instancias multilaterales como el Foro Global sobre Migración y Desarrollo (GFMD) (Hercog, y Siegler, 2010).

\section{La actuación económica}

Leclerc (2008) señala que la industria de tecnologías de la información le permitió a India hacerse un lugar en el escenario global. Además, desde mediados de los '80, con la apertura de una nueva etapa de outsourcing internacional, India se benefició con el asentamiento de centros de desarrollo de ultramar. Las redes de migrantes se destacaron en razón de que a las compañías multinacionales les resultaba mejor enviar empleados de origen indio para llevar adelante estos centros y por otro lado, los pioneros indios de esta fase, exitosos en Silicon Valley, que en su momento habían comenzado a invertir dinero en la apertura de negocios en India, podían asesorar a quienes recién llegaban, a través de distintas asociaciones (TIE, The Indus Entrepreneurs, Silicon Valley Indian Professional Association, etc.). De este modo y rápidamente, India se convirtió en el destino preferido de estas relocalizaciones de producción de software y servicios de tecnologías de la información, adquiriendo el país gran importancia también en la circulación global de profesionales de esta área.

Más allá de que la diáspora ha contribuido con la India a través de remesas, Inversión Extranjera Directa, transferencia de conocimiento y redes de emprendedores, Roy y Banerjee (2007) señalan que la Inversión Extranjera Directa (IED) no jugó en la India un rol tan importante como lo hizo en China y en tal sentido, sostienen que el Centro de Facilitación de los Indios de Ultramar (OIFC) desempeñará un papel crucial en reorientar el curso de los acontecimientos hasta ahora. Por otro lado, en términos de remesas, India supera a China, sin embargo, las remesas son menos

\footnotetext{
${ }^{17}$ De acuerdo a lo sostenido en su sitio web, el plan fue concebido durante la Conferencia Commonwealth sobre Asuntos Exteriores (Colombo, Ceylán, enero 1959) e iniciado en julio de 1951 como una espacio de cooperación para el desarrollo social y económico de las personas del Sur y Sureste de Asia (The Colombo Plan Secretariat, 2011) .

${ }^{18}$ ASEM es un foro interregional establecido en 1996, del que inicialmente participaban la Unión Europea, ASEAN, China, Japón, Corea y la Comisión Europea. Progresivamente fueron sumándose distintos miembros, entre ellos, India, en 2007, poseyendo en la actualidad 48 miembros (ASEMINFOBOARD, s. f.).
} 
eficientes que la Inversión Extranjera Directa en tanto sólo una fracción de las mismas es invertida (Kapur, 2004, citado en Chemouni, 2009).

Por su parte, Pandey, Aggarwal, Devane y Kuznetsov (2004), mencionan algunos de los grupos más exitosos dentro de la diáspora india, entre ellos: los migrantes de la zona de Gujarat en el este de África, que dominan sectores económicos claves como el de diamantes, los residentes indios en el Reino Unido, destacados en sectores de alta calificación; los indios en Estados Unidos, destacados también en distintas áreas y con un status material considerable, etc. ${ }^{19}$.

Ese papel de la diáspora en el ámbito económico está complementado por lo que Gayer y Therwath (2010) Ilaman una política contemporánea de ejemplaridad en Asia del Sur, en la que los modelos son mostrados como emprendedores cuya ejemplaridad es atestiguada por su éxito financiero. Los NRIs devinieron así, señalan, la síntesis de la "indianidad" y dieron cuerpo a la modernidad consumista y patriarcal, vistos como instrumentos de la modernidad en el país de origen al tiempo que factores del reconocimiento de la India como un poder internacional en occidente. Paralelamente, un sector cultural como el del cine Hindi popular, como señalaran Appadurai \& Brekenridge (1996, citados en Therwath, 2010), tuvo un rol central en la conformación de una identidad nacional y la promoción de un comportamiento normativo, también se destacó en esta etapa, mientras que en el presente, el cambio de gestión implicó cambios en los discursos, aunque la cultura sigue siendo un terreno de abono al soft power indio.

\section{La resonancia de construcciones políticas locales}

En cuanto a los ecos de la política "local"20 india en la diáspora, a modo de ejemplo, el inicio del siglo XX encuentra esos ecos políticos del país de origen en distintos lugares de asentamiento que se articularon con las circunstancias socio-políticas de los mismos. En el marco de consideración de estos sucesos, diremos que Baser (2010) retoma la idea del nacionalismo de larga distancia de Anderson (1983) y señala los dichos de Demmers (2001) acerca de que la mayor dificultad de asentamiento y asimilación en los países de residencia fortalecen la ligazón de las diásporas con sus países de origen. Mientras Sökefeld (2006) se refiere a las diásporas como comunidades transnacionales imaginadas, Adamson (2008) cree que el contexto de los movimientos sociales relacionados con una construcción social estratégica y la formación de identidad transnacional, podría ayudar a conocer mejor las razones de la movilización de las diásporas.

Si tomamos el caso de Estados Unidos a modo de ejemplo, las dos causas que organizaban en ese momento a los emigrados indios allí, eran la independencia india y la supresión de la discriminación racial en Estados Unidos. De hecho, se fundó el partido proindependista Ghadar (San Francisco, 1913), al tiempo que emigrados indios llevaban adelante acciones legales en el país por distintas causas (Guthikonda et al, 1979, citados en Therwath, 2007). Sen (2009) comenta la existencia, ya en 1912, del newsletter The Voice of Freedom, publicado en San Francisco y la existencia de textos en él que mostraban el fervor nacionalista, popular entre los indios allí expatriados.

La presencia de los emigrados indios en este país, atravesó por distintas circunstancias que

\footnotetext{
19 Para más información sobre la relación de la diáspora india con el desarrollo económico de India puede consultarse también: Newland, K. (2004); Anwar y Mughal (2009); Pradhan (2004, 2007, 2011), etc.

${ }^{20}$ Usamos "local" no con la idea de un concepto puro sino más bien como "una versión híbrida que combina procesos locales y globales en diferentes grados" (Oslender, s. f.).
} 
van desde la expulsión de Estados Unidos de más de 300 indios, en 1914, hasta la llegada al congreso como representante de California, de Dilip Singh Saund, en 1956 y el aumento en la población de este origen en ese país, especialmente tras 1965 (Guthikonda et al, 1979, citados en Therwath, 2007).

En los último tiempos, cobró notoriedad el eco político indio en la diáspora, del nacionalismo hindú, que alcanzara el gobierno a través del Partido Bharatiya Janata (BJP)21, en 1999, especialmente debido a las denuncias sobre el establecimiento de algunas organizaciones con ligazón financiera e ideológica con organizaciones en India que apoyan y difunden la política del hindutva ${ }^{22}$ (Ashraf, 2005). Al respecto, Therwath (2012) sostiene que el Sangh Parivar es el centro del movimiento hinduista - sea éste online u offline-, que el epicentro de las fuerzas nacionalistas hindúes en la diáspora se encuentra en los Estados Unidos de América (articulando un proyecto territorial con intereses universalistas en la web) y que allí, además, diferentes diásporas nacionalistas confluyen en la islamofobia ${ }^{23}$. En lo que respecta al ámbito online, señala también la existencia de una gran cantidad de sitios web relacionados al Sangh Parivar en otros países (Reino Unido, Países Bajos, etc.). Estas acciones del Sangh Parivar manifiestan una gran cohesión en esta red centralizada en India aunque cabe señalar que el accionar y propaganda de grupos pro-hindutva ${ }^{24}$ no representa a la mayoría de los migrantes hindúes en la diáspora, a pesar de que sus miembros se han establecido en puestos clave en las relaciones intercomunitarias e incluso las relaciones bilaterales (Therwath, 2012). Por lo demás, esos grupos conviven con el accionar de otros que luchan por el secularismo (Therwath, 2007).

Otros datos que señalan la implicancia de la diáspora en el escenario local indio son la expectativa de la diáspora de estar representada en el Parlamento a través de la Tercera Cámara (Dubey, 2009, citado en Hercog y Siegel, 2010) y la posibilidad dada a los NRIs de votar desde el exterior en las elecciones locales (The Times of India, 2012).

\section{Notas Finales}

Como señalamos, la diáspora india, es en la actualidad sujeto de otra mirada desde la gestión de gobierno en India y, esta ha tomado cada vez más acciones hasta llegar a la creación del Ministerio para los Asuntos de los Indios en el Extranjero a cargo de un ministro autónomo. En el centro de esta agencia nacional india sobre la diáspora aparece la figura de Gandhi, reivindicada en el Pravasi Bharatiya Diwas, quien tras estudiar en Londres fue a Sudáfrica, donde fue parte de la concreción de la creación del Congreso Indio de Natal, en 1894, previo advenimiento como figura central del nacionalismo indio.

\footnotetext{
${ }^{21}$ Mukta (1995) sintetiza que el BJP es un partido dedicado al reemplazo de la nación secular india por una basada en un nacionalismo hindú. Llegó al poder en Estados como Uttar Pradesh, Himachal Pradesh. Madhya Pradesh y Rajasthan, en 1991, teniendo como elemento simbólico en su plataforma la destrucción de la mezquita de Babri Masjid, en Ayodhya, Uttar Pradesh, cosa que concretaría tras su victoria, en 1992.

${ }^{22}$ El caso del Fondo Indio de Ayuda al Desarrollo (IDRF), creado en 1989 en Estados Unidos y señalado por sus nexos con el Sangh Parivar, al igual que sucedió con SEWA International, en el Reino Unido.

${ }^{23}$ Shah (2005) sostiene que el Rashtriya Swayamsevak Sangh (RSS) es extremo en cuanto a la hinduización del Islam en India y esa posición ha traído numerosos incidentes de violencia comunal en los que los musulmanes son las principales víctimas, que pierden sus vidas o resultan con sus propiedades dañadas. Esa violencia parece haber ido en aumento en años recientes, resultando, un caso testigo los ya mencionados incidentes en Bombay, en 1992, que derivaron en la destrucción de la Mezquita Babri en Ayodha y provocaron la muerte de alrededor de 2000 personas.
}

${ }^{24}$ El uso del término "hindutva" en este caso remite a la ideología del nacionalismo hindú. 
Vemos así que la búsqueda del compromiso de los emigrados, hoy bajo la figura de la diáspora india, implica el desarrollo de una tecnología que alude a un nacionalismo de larga distancia ${ }^{25}$ y el apoyo a la "madre patria" por parte de los migrantes, actuando ellos de acuerdo a los objetivos del país de origen. Además, la diferente concepción Nehruviana que une la "indianidad" a lo territorial y la de la derecha hindú (BJP) que la liga más a la etnicidad (pudiendo así la "indianidad" ser reivindicada por todos los miembros de la diáspora, en tanto respeten los valores hindúes), ha tornado el ámbito de la diáspora también en un espacio de disputa de estos significados. En el presente, con el nuevo gobierno, se continúa la política inaugurada por el BJP en cuanto a profundizar la religación de los emigrados con India y también con una "identidad India"26. Al respecto, mientras Leclerc (2004) se pregunta cuáles son las creencias que contempla esa identidad india, Khanna (2005), señala que:

...la India con su espléndidamente rica historia Hindu-Musulmana-Sikh,-BudistaCristiana-Judía-Jaina-Parsi es el "choque de civilizaciones que no fue" y con su secularismo, pluralismo tolerancia y diversidad (...) puede enseñar (...) una o dos cosas acerca de los llamados valores universales (Khanna, 2005: 10).

Estas políticas de articulación entre el Estado indio y su diáspora parecen desafiar la idea de que la globalización debilita al Estado nación y por el contrario fortalecer a este Estado (Chemouni, 2009), potenciando su soft power (Purushothaman, 2010).

Paralelo a ello, el desarrollo de un gran número de instituciones de migrantes indios basadas en diversas características, da cuenta de la manera en que la "diáspora" ha devenido un factor de autoreferencia e identificación política. Sin embargo, resulta necesario tener presente que no existen patrones homogéneos en la movilidad de personas como tampoco en sus procesos de adaptación en los lugares de residencia, en la evolución de sus identidades y/o en su religación con el país de origen.

Finalmente, al hablar de territorio, lo entendemos como el cruce entre una dimensión material/ geográfica en la que actúan las representaciones del espacio, determinando la comprensión del territorio como un espacio delimitado jurídicamente y otra social/simbólica en la que operan las prácticas espaciales inscriptas por los sujetos, es decir, los sentidos que los sujetos asignan al territorio. Hablamos así de la India entidad estatal/país de origen y de la India inserta en las redes e imaginarios transnacionales de la globalización, como un espacio nacional expandido. En relación a ello, Adamson (2008) señala que el término "diáspora", en mano de emprendedores políticos ha pasado de ser un descriptivo a prescriptivo, en cuanto categoría que sirve para organizar a los migrantes en formas de identificación, acción política, etc., y que así la diáspora se torna un efecto de procesos de movilización política, identidades y distintos imaginarios. En un sentido profundo entonces, se trata de cartografiar la movilidad, asignándole a los migrantes una continuidad con el espacio fundante, la Madre India ${ }^{27}$ y su historia, reinscribiendo el espacio y el tiempo, ambos

\footnotetext{
${ }^{25}$ Sobre nacionalismos de diáspora, se puede consultar Gellner (1988).

${ }^{26}$ De hecho, Malik y Metcalf (2011) plantean que existen tres nuevas fuentes de presión sobre el funcionariado diplomático indio: una clase de negocios ambiciosa, una diáspora que se hace oír y los medios de comunicación. Incluso sugieren que los Gobiernos interesados en profundizar sus relaciones bilaterales con India se acerquen a las comunidades de origen indio asentadas en sus territorios.

${ }^{27}$ Es dable señalar aquí que el objetivo de la reunión para la celebración del primer Pravasi Bharatiya Diwas, en 2003, fue "... reunir a la familia india de todo el mundo. También familiarizar a los indios con los logros de la diáspora india y usarlos como un puente
} 
conceptos políticos "en la forma en que las relaciones sociales están inscriptas y enmarcadas en ellos" (Oslender, 2002 [párr. 6]), "haciendo" a los migrantes parte de "una gran familia", potenciando las "raíces" y uniendo los "islotes del archipiélago". Ello deriva en nuevos centros y periferias ${ }^{28}$, atendiendo a que lo local y lo global formen parte de una relación mutuamente constitutiva, en la que los diversos elementos en juego deriven en distintos recursos y niveles de influencia para los agentes de tal relación.

\section{Referencias Bibliográficas}

-Adamson, F. B. Constructing the Diaspora: Diaspora Identity Politics and Transnational Social Movements. Ponencia presentada para el 49th Annual Meeting of International Studies Association, San Francisco, CA, (Marzo 26-29, 2008)

-Amrute, S. (2012). The New Non -Residents of India: A Short History of the NRI. D'Costa, A. P. - ed.A New India? Critical reflections in the Long Twentieth Century (pp. 127-150). UK \& USA: Anthem Press.

-Anwar, A. I. y Mughal, M. (2009). The role of Diaspora in attracting Indian Outward FDI. [consulta: 11 de enero 2011] .Recuperado de

https://editorialexpress.com/cgi-bin/conference/download.cgi?db_name=res_phd_2012\&paper $\underline{\mathrm{id}=208}$

-ASEMINFOBOARD, The Official Information Platform of The Asia - Europe Meeting (s.f.). [consulta: 11 de enero 2011]. Recuperado de http://www.aseminfoboard.org/about-asem-menu.html

-Ashraf, F. (2005). Indian Diaspora: Projecting Indian's image. Strategic Studies. Islamabad: Institute of Strategic Studies Islamabad (ISS). [consulta: 2 de mayo 2011] Recuperado de http://www.issi.org. pk/old-site/ss Detail.php?datald=331

-Baser, B. Stateless Diasporas and their long-distance nationalist activism in host countries. Ponencia presentada en el ECPR Fifth Pan-European Conference, Porto, Portugal (Junio, 23-26, 2010).

-Baumann, M. (2001). What You Always Wanted to Know About the Origins and Usage of That Word "Diaspora" or the Theology of Exile: Hope and Retribution. [consulta: 13 de mayo 2011]. Recuperado de http://www.irishdiaspora.net/

-Bhat, Ch., (1988). India and the Indian Diaspora. A Policy Issues. Occasional Paper 4. Department of Sociology. University of Hyderabag, [consulta: 17 de junio 2004]. Recuperado de http://www. indiandiaspora.nis.in/

-Bhat, Ch. Y Narayan, K. L. (2010). Indian Diaspora, Globalization and Transnational Networks: The South African Context. J Soc Sci, 25(1-2-3), 13-23.

-Cohen, R. (1997). Global Diasporas. An Introduction. London: UCL Press.

-Chaulia, S. S. (2002). BJP. India's Foreign Policy and the "Realist Alternative" to the Nehruvian para fortalecer las relaciones entre India y los países de residencia en esta era de la globalización".(Hindustan Times, 2002, citado en Sinha-Kerkhoff y Bal, 2003). Por lo demás, el Sumario Ejecutivo del evento indica: "The Pravasi Bharatiya Divas, the first of its kind involving the Indian Diaspora, was held in New Delhi, India from January 9th to 11th, 2003. It was organized by the Ministry of External Affairs, Government of India, along with the Federation of Indian Chambers of Commerce and Industry and created a successful consciousness of global Indian family among most participants and a message of togetherness of India and its Diaspora came out loud and clear.

This was the largest gathering of the global Indian family with 1,904 foreign delegates and 1,200 domestic delegates - a truly remarkable figure by any yardstick" (Indian Diaspora. First Pravasi Bharatiya Divas. January, 9-11, 2003).

${ }^{28}$ De hecho hubo una propuesta de la diáspora de establecer una "capital" de la diáspora, en Mauricio, atendiendo a la abrumadora presencia de personas de origen indio allí (60,35\% de la población) (MEA; 2002). 
Tradition. International Politics, 39, 215-234.

-Chemouni, B. (2009). The Diaspora as an economic asset: How China and India use their Diaspora to support their economic development. Tesis de grado. London School of Economics and Political Science. Londres: Inglaterra.

-Demmers, J. (2002). Diaspora and conflict: locality, long-distance nationalism, and delocalization of conflict dynamics. The public, 9, 1, 85-96.

-Dumont, G. F. (2008). Un nouvel acteur géopolitique: la Diaspora Indienne. Géostratégiques, 19 L'avenir géostratégique de la puissance indienne-, 39-53.

-Gamlen, A. (2006). Diaspora Engagement Policies: What are they, and what kinds of states use them? Working Paper n· 32. Oxford: COMPAS, University of Oxford. [consulta: 11 de junio 2004]. Recuperado de http://www.ssrc.org/workspace/images/crm/new_publication 3/\%7B0a6ab1518050-de11-afac-001cc477ec70\%7D.pdf

-Gayer, L. y Therwath, I. (2010). Introduction: Modeling Exemplarity in South Asia. South Asia Multidisciplinary Academic Journal (SAMAJ). Recuperado de http://samaj.revues.org/3011 -Gellner, E. (1988). Naciones y nacionalismo. Buenos Aires, Madrid : Alianza Editorial. -Hercog, M. and Siegel, M. (2010). Engaging the Diaspora in India. UNU-MERIT Working Paper Series. The Netherlands: Maastrich Graduate School of Governance, United Nations University. -Jayaram, N. (1998). The Study of Indian Diaspora: a multidisciplinary agenda”. Bangalore University. [consulta: 5 de mayo 2004] Recuperado de

http://www.uohyd.ernet.in/NJWORD1.htm

-Khadria, B. (2006). India: skilled migration to developed countries, labor migration to the Gulf. Migración y Desarrollo, 4-37. [consulta: 10 de septiembre 2009] Recuperado de http://meme.phpwebhosting.com/ migracion/modules/ve7/2.pdf -Khanna, P. (2008). What is Bollystan? Big Think. Recuperado de http://bigthink.com/ideas/2168 -Khanna, P. (2005). Bollystan: India's Diasporic Diplomacy. London: Foreign Policy Centre. [consulta: 14 de agosto 2009] Recuperado de ] Khanna Bollystan.pdf

-Leclerc, E. (2004). L'invention d'une diaspora indienne : enjeux politiques et sociaux. Ponencia presentada en "Espaces et sociétés aujourd'hui (la géographie sociale dans l'espace et dans l'action)", Rennes, UMR 6590, Université de Rennes (21-22 oct. 2004 ) [consulta: 11 de julio 2011] Recuperado de http://eso.cnrs.fr/lMG/pdf/le.pdf

-Leclerc, E. La Diaspora indienne: combien de divisions? Ponencia presentada en: Les Grands Thèmes du Festival International de Géographie. "Le monde en réseaux : lieux visibles, liens invisibles ». (30 de septiembre-2 de octubre, 2005) [consulta: 11 de julio 2011] Recuperado de http:// archives-fig-st-die.cndp.fr/actes/actes 2005/leclerc/article.htm

-Leclerc, E. (2008). India recentred: The role of Indian Diaspora in the globalisation process. Working Paper n- 2. IMDS Working Paper Series. International Migration and Diaspora Studies Project. Zakir Husain Centre for Educational Studies. School of Social Sciences. Jawaharlal Nehru University. New Delhi, India.

-Malik, A. y Metcalf, R. (2011). India's New World: Civil Society in the Making of Foreign Policy. Sydney: Lowy Institute for International Policy. [consulta: 7 de agosto 2011]. Recuperado de http:// www.lowyinstitute.org/publications/indias-new-world-civil-society-making-foreign-policy 
-MEA (Ministry of External Affairs). 2002. Report of the High Level Committee on Indian Diaspora. New Delhi: Indian Council of World Affairs.

-Mukta, P. (1995). The Politics of Religious Nationalism and New Indian Historiography: Lessons for the Indian Diaspora. Research Paper in Ethnic Relations n· 23. Coventry: Centre for Research in Ethnic Relations, University of Watwick.

-Narayan, K. L. (1998) Indian Diaspora: a demographic perspective. Occasional Paper N³, Centre for Study of Indian Diaspora. University of Hyderabag. [consulta: 17 de junio 2004]. Recuperado de http://www.uohyd.ernet.in/cinddiaspora/ocassional3.htm

-Newland, K. (2004). Beyond Remittances: The Role of Diaspora in Poverty Reduction in Countries of Their Origin. Washington, D. C.: Migration Policy Institute.

-Newland, K. (2010). Boice After Exit: Diaspora Advocacy. Migrants, Migration, and Development Program. Washington, D.C.: Migration Policy Institute.

-Nijhawan, S. (2003/4). Why has the Indian Diaspora been shunned by successive Indian governments? Thesis for MA International Studies and Diplomacy. School of Oriental and African Studies London, Faculty of Law and Social Sciences, Centre of South Asian Studies.

-Oonk, G. -ed.- (2007). Global Indian Diasporas: Exploring Trajectories of Migration and Theory. Amsterdam: Amsterdam University Press.

-Oslender, U. (2002). Espacio, lugar y movimientos sociales: hacia una 'espacialidad de resistencia. Scripta Nova. Revista electrónica de Geografía y Ciencias Sociales. Universidad de Barcelona. Vol. VI, Nro. 115, junio.

-Oslender, U. Espacializando resistencia: perspectivas de "espacio y "lugar" en las investigaciones de los movimientos sociales. Biblioteca Luis Ángel Arango. Banco de la república. Colombia. [consulta: 19 de octubre 2009] .Recuperado de

\section{http://www.banrep.gov.co/blaavirtual/letra-o/osle/pres.htm}

-Pandey, A.; Aggarwal, A; Devane, R. y Kuznetsov, Y. (2004). India's Transformation to Knowledgebased Economy - Evolving Role of the Indian Diaspora. Evalueserve. [consulta: 4 de junio 2009].

Recuperado de http://info.worldbank.org/etools/docs/library/152386/abhishek.pdf

-Pradhan, J. (2004). The Determinants of Outward Foreign Direct Investment: A Firm Level Analysis of Indian Manufacturing. Oxford Development Studies, 32, 4, 619-639.

-Pradhan, J. (2007). Trends and Patterns of Overseas Acquisitions by Indian Multinationals. New Delhi: ISID Working Paper 2007/10.

-Pradhan, J. (2011). Emerging Multinationals: A Comparison of Chinese and Indian Outward Foreign Direct Investment. International Journal of Institutions and Economies, 3, 1, 113-148.

-Purushothaman, U. (2010). Shifting Perceptions of Power: Soft Power and India's Foreign Policy. Journal of Peace Studies, 17, 2\&3. Recuperado de http://www.icpsnet.org/adm/pdf/1291714915.pdf -Roy, J. y Banerjee, P (2007). Attracting FDI from the Indian Diaspora: The Way Forward. [consulta: 4 de junio 2009]. Recuperado de http://www.oifc.in/Uploads/MediaTypes/Documents/ AttractingFDIFromlndianDiaspora.pdf

-Sen, A. (2009). Past Knowing and representational Practices: Visual Culture and the Social Production of Peoplehood. Twenty-First Century Papers n-11. Milwaukee: University of Wisconsin.

-Shah, S. A. A. (2005). Religious Terrorism in Other Faiths. Strategic Studies. Islamabad: Institute of Strategic Studies Islamabad (ISS). [consulta: 22 de mayo 2011]. Recuperado de http://www.issi.org. 
pk/old-site/ss Detail.php?datald=348

-Sharma, J. (s.f.) Características de la diáspora india y su relación con el país de origen. [consulta: 27de febrero 2010]. Recuperado de http://rimd.reduaz.mx/coleccion_desarrollo migracion/relaciones-estado1/RelacionesEstado1_3car acteristicas.pdf

-Sheffer, G. (1986). Modern Diasporas in International Politics. Australia: Croom Helm Ltd.

-Sinha-Kerkhoff, K y Bal, E. (2003). Eternal Call of the Ganga. Reconnecting with People of Indian Origin in Surinam. Economic and Political Weekly, 2003, 38, 38, 4008-4021.

-Terrazas. A. (2008). Indian Immigrants in the United States. Washington: Migration Policy Institute. [consulta: 4 de octubre 2009]. Recuperado de http://www.migrationinformation.org/usfocus/display. cfm?ID=687

-Therwath, I. (2007). Working for India or against Islam? Islamophobia in Indian American Lobbies. South Asia Multidisciplinary Academic Journal (SAMAJ). [consulta: 4 de octubre 2010]. Recuperado de http://samaj.revues.org/262

-Therwath, I. (2010). 'Shining Indians': Diaspora and Exemplarity in Bollywood. South Asia Multidisciplinary Academic Journal (SAMAJ). [consulta: 12 de septiembre 2010]. Recuperado de http://samaj.revues.org/3000

-Therwath, I. (2012). Cyber-Hindutva: Hindu Nationalism, the diaspora nad the web. New Delhi: Centre de Sciences Humaines.

-The Colombo Plan Secretariat. [consulta: 4 de octubre 2011]. Recuperado de http://www.colomboplan.org/

-The Indian Diaspora (High Level Committee on Indian Diaspora). [consulta: 4 de octubre 2011]. Recuperado de http://indiandiaspora.nic.in/contents.htm

-The Indian Diaspora. First Pravasi Bharatiya Divas. January, 9-11, 2003. [consulta: 4 de octubre 2011]. Recuperado de http://indiandiaspora.nic.in/ch1.pdf

-The Times of India. (2012, Enero, 9). NRIs can vote in Indian elections: Manmohan. [consulta: 11 de marzo 2012] . Recuperado de

http://timesofindia.indiatimes.com/nri/specials/pravasi-bharatiya-divas/pravasi-bhartiya-news/NRIscan-vote-in-Indian-elections-Manmohan/articleshow/11417075.cms

-Varadarajan, L. (2010). The Domestic Abroad. Diasporas in International relations. New York: Oxford University Press.

-Vertovec, S. (1999). Three meanings of "diáspora", exemplified among South Asian religions. Diaspora, 7, 2.

-Vertovec, S. y Cohen, R. (1999). Migration, Diasporas and Transnationalism. Massachusetts: Edward Elgar Publishing Limited. 